\title{
Antennas For Satellite Communication
}

\author{
Viplav Yadav*1, Sandeep², sanjay sharma ${ }^{3}$
}

*Corresponding Author: -

\begin{abstract}
: -
Satellite communication antenna in unvield.It also comprises of transmitting/ receiving signal section and transmission of signal to satellite. it consist of various types of antennas used in satellite communication such as $U H F, L / s$ band,cband, ku-band, k-band antennas. Multibeam broadband antenna and antennas for geostationary for satellite communication is also explained in this paper. This paper mainly describes the the signal transmitting/receiving antennas for satellite communication.
\end{abstract}

\section{(ㄷ) (\$) (1)}




\section{INTRODUCTION}

The antenna is the most visible part of the satellite communication system. The antenna transmits and receives the modulated signal at the radio frequency portion of the electromagnetic spectrum. For satellite communication, the frequencies range about $0.3 \mathrm{Ghz}(\mathrm{VHF})$ to $30 \mathrm{Ghz}$ (ka-band) and beyond [1]. These frequencies represent microwaves, with wavelength on the order of one meter down to below one centimeter. High frequencies and the corresponding small wavelength, permit the use of antennas having practical dimensions for commercial use. this article summarizes the basic properties of antennas used in satellite communication and derives several fundamental relations used in antenna design and RF link analysis [1].

\section{UHF Satellite Communications Antennas}

The AV 2040 is a foldable, manpack, high-gain, UHF Satellite Communication (SATCOM) antenna designed for special missions where portability and high gain are required.

\begin{tabular}{ll} 
Frequency & $240-400 \mathrm{MHz}$ \\
\hline VSWR & $1.5: 1$ \\
\hline Polarization & Right hand, circular \\
& AV 2040-3: +7.0 dBic, 240-400 MHz \\
& AV 2040-2 +11.0 dBic, 240-318 MHz \\
Gain & AV 2040-1 +9.0 dBic, 240-400 MHz \\
\hline Power Handling & 200 Watts CW \\
\hline Axial Ratio & $3 \mathrm{~dB}$ \\
\hline Weight & $6.5 \mathrm{lbs}$ \\
Connector Type & BNC Male \\
\hline Finish & Black \\
\hline Military P/N & AV2040-3 \\
\hline
\end{tabular}

\section{L/S-band Mobile Satellite Communications Antennas}

The present invention relates to satellite communications systems. More particularly, the present invention relates to satellite communications systems providing Internet service to computer users[2].

Computer networking is rapidly becoming ubiquitous in present day society. Computer networks have grown from isolated connections among research scientists and universities to the "information superhighway" of today. Access to this superhighway is typically accomplished through the "Internet". The Internet utilizes a transmission control protocol/Internet protocol (TCP/IP) communications system which is utilized by hundreds of millions of users worldwide[2].

Through the use of standardized formats, computer users can easily access and navigate through the abundance of information which is accessible through the Internet. This information includes not only research and news information but has now spread to commercial advertising and business communications which permit users to download images, video, sound clips and/or text documents relating to a company's products. This information is transmitted over the Internet in measurements commonly referred to as bits.

\section{C-, Ku- and Ka-band FSS/BSS Antennas C band}

The $\mathbf{C}$ band is a name given to certain portions of the electromagnetic spectrum, including wavelengthsof microwaves that are used for long-distance radio telecommunications. The IEEE C-band (4 to $8 \mathrm{GHz}$ ) - and its slight variations - contains frequency ranges that are used for many satellite communications transmissions, some Wi-Fi devices, some cordless telephones, and some weather radar systems. For satellite communications, the microwave frequencies of the $\mathrm{C}$-band perform better under adverse weather conditions in comparison with $\mathrm{K}_{\mathrm{u}}$ band $(11.2 \mathrm{GHz}$ to $14.5 \mathrm{GHz})$ microwave frequencies, which are used by other communication satellites. ${ }^{[1]}$ The adverse weather conditions, collectively referred to as rain fade, all have to do with moisture in the air, including rain and snow.

Ku

$\mathrm{Ku}$ band is primarily used for satellite communications, most notably for fixed and broadcast services, and for specific applications such as NASA's Tracking Data Relay Satellite used for both space shuttle and International Space Station(ISS) communications. Ku band satellites are also used for backhauls and particularly for satellite from remote locations back to a television network's studio for editing and broadcasting. The band is split into multiple 
segments that vary by geographical region by the International Telecommunication Union (ITU). NBC was the first television network to uplink a majority of its affiliate feeds via $\mathrm{Ku}$ band in 1983.

\section{Ka band}

The Ka band (Pronounced: "Kay-Ay Band") covers the frequencies of $26.5-40 \mathrm{GHz},{ }^{[1]}$ i.e. wavelengths from slightly over one centimeter down to 0.75 centimeters. ${ }^{[2]}$ The $\mathrm{Ka}$ band is part of the $\mathrm{K}$ band of the microwave band of the electromagnetic spectrum. This symbol refers to "K- above" — in other words, the band directly above the K-band. The $30 / 20 \mathrm{GHz}$ band is used in communications satellites, uplink in either the $27.5 \mathrm{GHz}$ and $31 \mathrm{GHz}$ bands, ${ }^{[3]}$ and highresolution, close-range targeting radars aboard military airplanes. Some frequencies in this radio band are used for vehicle speed detection by law enforcement. ${ }^{[4]}$ Kepler Mission uses this frequency range to downlink the scientific data collected by the space telescope.

\section{Multibeam Broadband Satellite Communications Antennas}

This invention relates to the field of antenna systems for communicating between an earth station and a satellite. More particularly, it relates to a reflector for use in an antenna system and to an antenna system using a plurality of such reflectors for broadband satellite communications systems operating in the microwave and millimeter wave frequency bands. The invention further relates to antennas for communicating with low- and mid-earth orbiting satellites, which traverse the sky somewhat rapidly[3].

In past decades, there have come into widespread use communications systems which employ earth-orbiting satellites to relay communications between earth-based stations ("satellite communications systems"). Now under development and in early stages of deployment are satellite communications systems which utilize broadband signaling and operate in the 11-14 GHz (Ku) band, the 20-30 GHz (Ka) band, and higher millimeter wave bands between about 30 and $70 \mathrm{GHz}$. (Such systems are hereafter referred to as MMW systems or as $\mathrm{Ku}, \mathrm{Ka}$ or $\mathrm{V}$ band systems.) Many of these MMW systems employ low-earth-orbit (LEO) or mid-earth-orbit (MEO) satellites in constellations, to provide bi-directional, high-speed data links from and to customer premises equipment (CPE) located at various places. They may also use one or more geostationary satellites (GEO's) in combination with LEO or MEO satellites in some types and modes of communications. The Spaceway, Expressway, Cyberstar, and Teledesic systems are the most well-known of these new systems; but in fact there are more than twenty MMW satellite systems in various stages of development and implementation.

\section{Antennas for Non-geostationary Constellations}

Geostationary ("geo") satellites for telecommunications applications were first proposed many years ago by the author Arthur C. Clark. Today, there are numerous communications systems employing geo satellites for such diverse applications as telephone and data trunking, television distribution, direct-to-home broadcasting, and mobile communications. Geo satellites operate on the physical principle that a satellite, in circular orbit at the proper altitude above the equator, will orbit the earth at the same angular velocity as the earth's rotation. These satellites therefore, appear to be fixed relative to a point on the earth. This characteristic of geo satellites facilitates their use for communications applications by allowing communications terminals on the earth to simply point their antennas at essentially one position in the sky.[3]

\section{Advanced technology in satellite communication antennas: Electrical and mechanical design}

Antenna technology for earth stations and satellites is discussed. The fundamentals, including common analysis methods, for antenna technology for Satellite Communication are reviewed. Details of the analysis method for beam waveguide feeds are shown, and electrical and mechanical designs for earth station antennas are examined. Offset dual-reflector .antennas and offset reflector .antennas with beam waveguide feed are examined, giving comprehensive descriptions of mechanical techniques. The homology design technique for very large and precise antennas and the holographic measurement method of reflector surface error are discussed. Shaped-beam and multibeam antennas are addressed, as is product assurance.[4]

\section{References}

[1].http://onlinelibrary.wiley.com/doi/10.1002/9781119945147.ch12/summary

[2].http://ocw.mit.edu/courses/aeronautics-and-astronautics/16-851-satellite-engineering-fall-2003/lecturenotes/121satelitecomm2_done.pdf

[3].http://personal.ee.surrey.ac.uk/Personal/L.Wood/publications/MSc-thesis/wood-msc- thesis.pdf

[4].http://ultralifecorporation.com/commsys/products/adapters-and-accessories/av-2040-uhf-satellite-communicationsatcom-antenna-system/ 